\title{
Reliability Analysis of Mechanical Products Based on Regenerative Samples
}

\author{
Tengfei Chen ${ }^{a^{*}}$, Erling Gong ${ }^{b}$ \\ Mechanical engineering and automation, National University of Defense Technology, Changsha, \\ 410073, China \\ a*13278893156@163.com, byongerling@sina.com
}

Key words: Mechanical products; small sample; time censoring; Bootstrap; BP neural network

\begin{abstract}
Aiming at the problem of reliability estimation of mechanical products in small sample size and time censored test under Weibull distribution, this paper proposed a method which combined Bootstrap and BP neural network for reliability evaluation. Firstly, Bootstrap method was used to expand the reliability and failure time sample. Secondly, the BP neural network was trained by the expanded sample. Then the parameters of Weibull distribution can be estimated by the trained BP neural network. Finally, the reliability characteristics of the product can be obtained. In the end, an example was analyzed to illustrate the applicability of the method.
\end{abstract}

\section{Introduction}

With the development of science and technology, the performance parameters of mechanical products are increasing day by day, the applicable locations are more extensive, and the application environment is becoming worse, so the problem of product performance and reliability is more and more outstanding ${ }^{[1]}$. However, due to the limitations of research and development cost and test conditions, a large number of tests can't be carried out for the product. Time censoring is the most commonly used method ${ }^{[2]}$. Therefore, in the reliability evaluation of mechanical products, evaluation theory of small sample is the main method, and it has important practical significance to study the reliability evaluation of small samples.

At present, the reliability evaluation methods of small sample test mainly include the following methods: semi empirical method, Bayes method, Bootstrap method, virtual augmented sample evaluation method and so on. Among them, the core idea of Bootstrap method and virtual augmented sample evaluation method is sample regeneration ${ }^{[3]}$. In the field of sample regeneration methods, a great deal of contributions have been made by domestic scholars. Aiming at the extremely small sample problem in the reliability evaluation of mechanical products in aviation field, Fang Ya ${ }^{[4]}$ put forward the extreme small sample reliability evaluation method based on virtual augmented sample extension method. The solution to the life test data of $n=2$ and $n=3$ in engineering is put forward. Finally, the rationality and validity of the method are proved by a case study. Gao Pandong ${ }^{[5]}$ proposed a new method of reliability evaluation based on Weibull distribution and small sample data. According to the information of the sample itself, B-MUME method expands the sample through the principle of self-sampling. The parameters of Weibull distribution can be determined by calculating the regenerative samples of each group through MUME method. Then the reliability parameter estimation of the product is obtained. Ding Fei ${ }^{[6]}$ proposed a method that when the censored data are small samples the BP neural network model can be used to learn and simulate for sample regeneration. Then the parameters of Weibull distribution are estimated by regenerative sample.

In the case of small samples, the effective way to improve the accuracy of reliability evaluation is to increase the amount of information. In this paper, a parameter estimation method of two parameter Weibull distribution based on bootstrap method is proposed. BP neural network model is used to train the regenerated samples. The accuracy of the reliability characteristics can be improved. 


\section{Basic Knowledge}

\subsection{Two Parameter Weibull Distribution Model And Parameter Estimation}

In the assessment of reliability, Weibull distribution is one of the most widely used distributions in life data analysis. By adjusting the distribution parameters, a variety of distributions can be constructed to establish models for various life distribution characteristics of different engineering projects. The life of most mechanical products and equipment is subject to the Weibull distribution. The probability density function of two parameter Weibull distribution is:

$$
f(t)=\frac{\beta}{\eta}\left(\frac{t}{\eta}\right)^{\beta-1} e^{\left[-\left(\frac{t}{\eta}\right)^{\beta}\right]}
$$

$\beta$ is the shape parameter, and $\eta$ is the scale parameter. When the variable of two parameter Weibull distribution is life $t$, its reliability function is:

$$
R(t)=1-F(t)=e^{-\left(\frac{t}{\eta}\right)^{\beta}}
$$

In the failure analysis of the product, $\beta$ is related to the failure mechanism of the product, and different values are accompanied by different failure mechanisms ${ }^{[7]}$. The reliability function $R(t)$ shows that the mechanical product life and reliability of the two parameter Weibull distribution is determined by $\beta$ and $\eta$. The linear transformation is used to estimate the parameters of Weibull distribution in reference [6].

\subsection{Bootstrap Method And Resampling Process}

On the basis of summarizing and generalizing previous research results, Professor Efron ${ }^{[8]}$ of Department of Statistics at Stanford University proposed a new statistical inference method (Bootstrap) in the United States in 1979. The purpose of the Bootstrap method is to use the existing samples to imitate the unknown distribution. And the method can take full advantage of the information of the sample itself, and do not need to make assumptions about the overall distribution. In engineering practice, Bootstrap method is an effective way to overcome the difficulties of data limitation.

The mathematical description of the Bootstrap method is as follows: the random sample $X=$ $\left[x_{1}, x_{1}, \cdots x_{n}\right]$ comes from the unknown population distribution $F$. Select a random variable $R(X, F)$ which is the function of $X$ and $F$. Then the distribution characteristics of $R(X, F)$ can be estimated based on the random sample $X$. The essence of Bootstrap is a process of resampling. The concrete procedures are in reference [8].

\subsection{BP Neural Network Model}

Rumelhart and McClelland proposed the BP algorithm in 1985, and BP neural network is a multilayer feedforward network trained by the error propagation algorithm. BP neural network can learn and store a large number of input-output schema mappings without requiring knowledge of the mathematical equations of this mapping in advance. Based on this feature, the BP neural network is used to learn and simulate the sample data, and establish the input-output mapping relationship, finally achieve the approximation of the function ${ }^{[9]}$. BP neural network can be implemented through Matlab neural network toolbox. By adjusting the number of hidden layer and training algorithm, we can find a neural network model which is more suitable for the current sample. A typical 3 layer BP neural network model is shown in figure 1.

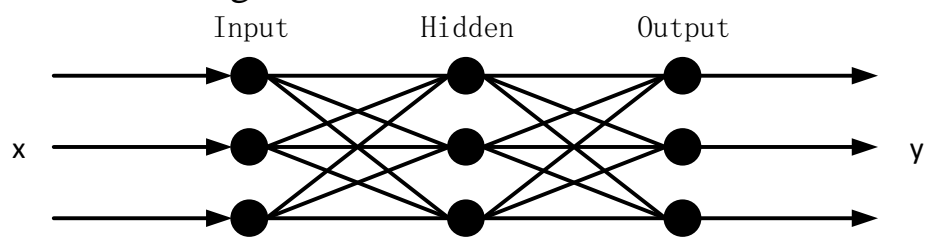

Fig. 1 Three layer BP neural network model structure

In order to reduce the simulation error, 3 layer neural network is selected. In addition, the Levenberg-Marquardt algorithm combines Gauss Newton algorithm and steepest descent method. L$\mathrm{M}$ algorithm has local convergence of Gauss Newton algorithm and the global characteristics of 
steepest descent method, and has faster iterative convergence speed. Therefore, L-M algorithm is chosen as the training algorithm of neural network.

\section{Data Processing Method of Time Censored Test}

To estimate the parameters of the Weibull distribution with the time censored test data, there are two methods: graphical and analytical methods. In this paper, the graphical method is used to analyze the fault data of Weibull distribution. The empirical distribution graph method in graphical method is easy to use and can guarantee a certain accuracy ${ }^{[10]}$. According to a large number of statistical tests, when the sample size is small, the empirical reliability can be calculated by the median rank formula. The median rank formula is:

$$
R\left(t_{i}\right)=1-\frac{i-0.3}{n+0.4}(i=1,2, \cdots, n)
$$

$R\left(t_{i}\right)$ is the median rank estimated value of empirical reliability, $t_{i}$ is the running time of the $i$ th failure sample; $i$ is the failure sample number; $N$ is the total sample number.

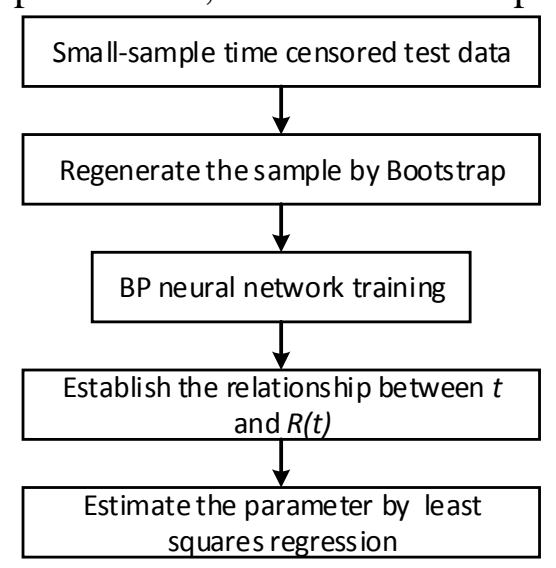

Fig. 2 Bootstrap-BP neural network method

It is difficult to guarantee the accuracy of parameters because the time censored data are small samples. In this paper, on the basis of regenerating the censored samples with the Bootstrap method, the BP neural network is trained to establish the relationship between $t$ and $R(t)$. Then the least squares regression is used to estimate the parameters, and the parameters with higher precision are obtained. The implementation process is shown in figure 2.

\section{Case Study}

A time censored test of 44 mechanical products produced by a company was carried out. The censored time is $1100 \mathrm{~h}$. And there were 9 products that fail in $1100 \mathrm{~h}$. The failure time are: 666.4 , 704.2, 742.8, 766.5, 785.5, 792.1, 850.3, 934.4, 1058.5. The unit of time are hours (h). The following table can be established according to the method described in this paper.

Table 1. Time censored test data

\begin{tabular}{|c|c|c|c|}
\hline Number of Fail & $\begin{array}{c}\text { Time of Fail } \\
(t)\end{array}$ & $\begin{array}{c}\text { Estimation of } F\left(t_{i}\right) \\
(i-0.3) /(N+0.4)\end{array}$ & $\begin{array}{c}\text { Estimation of } \mathrm{R}\left(t_{i}\right) \\
1-F(t i)\end{array}$ \\
\hline 1 & 666.4 & 0.016 & 0.984 \\
\hline 2 & 704.2 & 0.038 & 0.962 \\
\hline 3 & 742.8 & 0.061 & 0.939 \\
\hline 4 & 766.5 & 0.083 & 0.917 \\
\hline 5 & 785.5 & 0.106 & 0.894 \\
\hline 6 & 792.1 & 0.128 & 0.872 \\
\hline 7 & 850.3 & 0.151 & 0.849 \\
\hline 8 & 934.4 & 0.173 & 0.827 \\
\hline 9 & 1058.5 & 0.196 & 0.804 \\
\hline
\end{tabular}

The Bootstrap-BP neural network method proposed in this paper is implemented as follows:

(1) According to the time censored test data, the Bootstrap method is used to regenerate the failure 
time and empirical reliability. Take $N=10000$, then the extended data of 1000 sets of samples with data of 9 were obtained, and the data were reconstructed to get the data of sample size of 90000;

(2) The BP neural network model is trained by the large sample data we got, and the relationship between $t$ and $R(t)$ can be obtained;

(3) Randomly generating a set of data close to the empirical distribution data, such as 100 random numbers in $[0.8,1]$. The generated random number is used as the input of the neural network, and the new sample data is obtained by the neural network simulation;

(4) The parameters of Weibull distribution are obtained by the least squares fitting of the sample data.

According to the steps above, the following estimation results can be obtained by Bootstrap, BP neural network and Bootstrap-BP neural network respectively.

Table 2. Parameter estimation results of Weibull distribution

\begin{tabular}{|c|c|c|c|c|}
\hline The method & Fitting formula & $\begin{array}{c}\text { Shape } \\
\text { parameter } \beta\end{array}$ & $\begin{array}{c}\text { Scale } \\
\text { parameter } \eta\end{array}$ & $\begin{array}{c}\text { Interval estimation of } \\
\beta\end{array}$ \\
\hline Direct fitting & $\begin{array}{l}\mathrm{y} \\
=5.1661 \mathrm{x} \\
-36.9745\end{array}$ & 5.1661 & 1283.2 & {$[0.4312,15,7855]$} \\
\hline Bootstrap & $\begin{array}{l}\mathrm{y}=5.2334 \mathrm{x} \\
-37.3299\end{array}$ & 5.2334 & 1252.6 & {$[0.3848,14.0850]$} \\
\hline BP neural network & $\begin{array}{l}\mathrm{y}=5.6314 \mathrm{x} \\
-40.1981\end{array}$ & 5.6314 & 1259.2 & {$[0.4233,15.4935]$} \\
\hline $\begin{array}{c}\text { Bootstrap-BP neural } \\
\text { network }\end{array}$ & $\begin{array}{l}\mathrm{y}=5.9476 \mathrm{x} \\
-42.2453\end{array}$ & 5.9476 & 1215.6 & {$[0.3075,11.2549]$} \\
\hline
\end{tabular}

\section{Conclusion}

The Bootstrap-BP neural network method proposed in this paper makes full use of the information of the sample itself. It can realize the data regeneration, enlarge the sample size, and make up the shortcomings of small sample information.

Bootstrap-BP neural network method combined Bootstrap and BP neural network. The problem that training samples are insufficient when using BP neural network alone is solved. To a certain extent, the problem that the value range of the regenerative samples is limited in Bootstrap method is improved. By testing the confidence interval of the shape parameter of Weibull distribution, this method can get a shorter interval estimate. This proves the validity and correctness of the proposed method in the reliability evaluation of small sample mechanical products.

\section{References}

[1]. Xu Weibao, Zhong Tao. Reliability design and test of mechanical product [M]. National Defense Industry Press, 2015. (In Chinese)

[2]. Sirvanci M, Yang G. Estimation of the Weibull parameters under type I censoring [J]. Journal of the American Statistical Association, 1984, 79 (385):183-187.

[3]. Feng Yunwen, Huang Wei, Lv Zhenyu. Semi empirical evaluation method for very small sample test [J]. Journal of Aeronautics, 2004, 25 (5):456-459. (In Chinese)

[4]. Fang Ya. Research on reliability evaluation method of mechanical products [D]. Northwestern Polytechnical University, 2007. (In Chinese) 
[5]. Gao Pandong, Shen Xuejin, Chen Xiaoyang, Xia Xintao. Reliability analysis under Weibull distribution in small samples based on Bootstrap [J]. Machine Design and Research, 2015, 31 (2):164-167. (In Chinese)

[6]. Ding Fei, Jin Xin, Wang Chunhua. Evaluation on reliability of hydraulic powered support under small sample event [J]. Coal Science and Techology, 2016, 44 (11):116-120. (In Chinese)

[7]. Song Qi, Feng Guodi, Xue Lu. Research on fault rate distribution and reliability evaluation of a kind of earthwork machinery product [J]. Construction Machinery, 2015, 46 (10):33-39.

[8]. Efron B. Bootstrap Method [J]. Ann, Statist, 1979, (7):1-36.

[9]. Elhewy A H, Mesbahi E, Pu Y. Reliability Analysis of Structure Using Neural Network method [J]. Probabilistic Engineering Mechanics, 2006, 21 (1):44-53.

[10]. Bhattacharya P, Bhattacharjee R. A Study on Weibull Distribution for Estimating the Parameters [J]. Wind Engineering, 2011, 33 (5):469-476. 